\title{
Naphthylethylamines as chiral auxiliaries in a stereoselective formation of trans- $\beta$-lactams via Staudinger cycloaddition
}

\author{
Vanya B. Kurteva, ${ }^{a}$,* Aleksandar R. Todorov, ${ }^{a}$ and Margarita Stoylova-Dicheva ${ }^{b}$ \\ ${ }^{a}$ Institute of Organic Chemistry with Centre of Phytochemistry, Bulgarian Academy of Sciences, \\ Acad. G. Bonchev str., bl. 9, 1113 Sofia, Bulgaria \\ ${ }^{b}$ Institute of Microbiology, Bulgarian Academy of Sciences, Acad. G. Bonchev str., bl. 26, 1113 \\ Sofia, Bulgaria \\ E-mail:vkurteva@orgchm.bas.bg
}

\begin{abstract}
Dedicated to our colleague and friend Prof. DSc Petko Ivanov on the occasion of his $65^{\text {th }}$ anniversary
\end{abstract}

\begin{abstract}
As a part of our study on the chiral amine induced stereoselectivity in the formation of the transazetidin-2-one ring via Staudinger cycloaddition, the efficiency of the enantioisomeric naphthylethylamines as chiral auxiliaries was examined. It was found that the isomeric 1-(2naphthyl)ethylamines induced commensurable selectivities with those obtained by the corresponding phenylethylamines, good to excellent yields with de up to 48\%. In contrast, the 1(1-naphthyl)ethylamine auxiliaries led to excellent conversion but with dramatic loss of selectivity, with a maximum of $14 \%$ de. The $\beta$-lactams obtained were screened in vitro against 10 bacteria and 3 fungi but significant antimicrobial activities were not observed.
\end{abstract}

Keywords: Chiral trans- $\beta$-lactams, Staudinger cycloaddition, chiral naphthylethylamine auxiliaries, diastereoselectivity, antimicrobial activity

\section{Introduction}

Compounds possessing the $\beta$-lactam skeleton are of great importance as they constitute one of the most successful classes of therapeutic agents to date. ${ }^{1}$ The family of $\beta$-lactam antibiotics, which is among the most widely employed antimicrobial agents, includes numerous representative sub-groups, differing in their structural elements as well as in the azetidinone ring stereochemistry. ${ }^{2}$ For instance, the penicillin and cephalosporin antibiotics possess $c i s$ - $\beta$-lactam units, whereas thienamycins and trinems have trans- $\beta$-lactam moieties. Thienamycins are carbapenems of special interest as they display high activity against bacteria that are resistant to other $\beta$-lactamase stable molecules. ${ }^{3}$ The most commonly prescribed for medicinal consumption 
are the derivatives of thienamicin imipenem, ${ }^{4}$ meropenem, ${ }^{5}$ ertapenem, ${ }^{6}$ doripenem, ${ }^{7}$ which are stable in aqueous solution, hence overriding the impracticability of the parent compound. 2Azetidinones are also versatile building blocks for the stereoselective synthesis of various non- $\beta$ lactam products. ${ }^{8}$

Considerable effort has been devoted to the construction of azetidinone ring resulting in the development of numerous synthetic protocols. ${ }^{9}$ The asymmetric variants, ${ }^{10}$ involve application of chiral organometallic catalysts as well as non-catalytic transformations. Among the latter, chiral auxiliary induced enantioselective Staudinger [2+2] ketene-imine cycloaddition is regarded as one of the most fundamental and versatile methodologies for the synthesis of chital $\beta$-lactams. The majority of the protocols have exploited chiral ketene precursors, ${ }^{11}$ some induced excellent selectivities. However, these approaches generally suffer from such drawbacks as low yields, difficult to obtain starting materials or tedious workup procedures. The efficiency of chiral amine auxiliaries, one of the cheapest chiral sources on the market, has been poorly studied, mainly in the synthesis of cis-azetidinones, ${ }^{12}$ while only few records describe the formation of transcompounds. ${ }^{13}$

As a continuation of our study on the stereoselective formation of trans- $\beta$-lactams via Staudinger cycloaddition induced by chiral amine auxiliaries, we present herein the results on the efficiency of enantiomeric 1-(2-naphthyl)ethylamines and 1-(1-naphthyl)ethylamines. The chiral azetidinones obtained are screened for antimicrobial activity.

\section{Results and Discussion}

The trans-azetidinones 4-15 were obtained as previously described ${ }^{13 \mathrm{c}}$ in order to compare the stereoselectivity of the transformation caused by naphthylethylamine (1) auxiliaries with that of the corresponding phenylethylamines. The set of aromatic aldehydes (2), which led to efficient conversion and selectivity in the phenyl series, was used. Namely, compounds possessing electron donating substituents, which are expected to slow the direct closure and to accelerate the isomerization of the zwitterionic intermediate, 4-dimethylamino-, 3,4-dimethoxy-, and 2,4,6trimethoxybenzaldehyde. The representative protocol, shown on Scheme 1, was as follows: a) intermediate imines $\mathbf{3}$ were obtained by irradiating mixtures of an amine $\mathbf{1}$ and aldehyde $\mathbf{2}$ in a microwave oven with a power of $800 \mathrm{~W}$. b) Staudinger [2+2] ketene-imine cycloaddition was carried out in refluxing toluene or xylene by using 3-phenylpropionyl chloride as a ketene precursor and triethylamine as a base. 


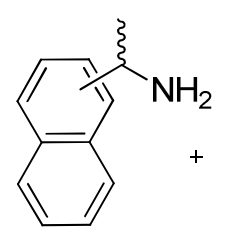

1<smiles>[R]c1ccc(C=O)cc1</smiles>

2

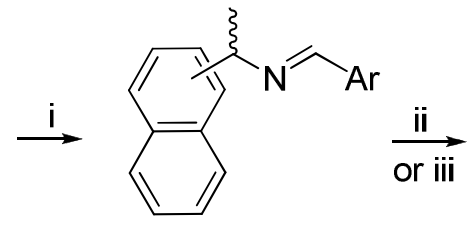

3a-3I

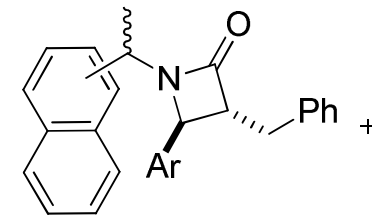

$(3 R, 4 S)-4-15$

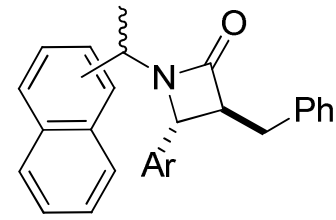

$(3 S, 4 R)-4-15$

\begin{tabular}{c|ccc} 
amine 1 & $\mathrm{R}-\mathrm{Me}_{2} \mathrm{~N}$ & $3,4-(\mathrm{MeO})_{2}$ & $2,4,6-(\mathrm{MeO})_{3}$ \\
\hline (S)-1-(2-naphthyl)ethylamine & $\mathbf{3 a} / \mathbf{4}$ & $\mathbf{3 b} / \mathbf{6}$ & $\mathbf{3 c} / \mathbf{8}$ \\
$(R)-1-(2-$ naphthyl)ethylamine & $\mathbf{3 d} / \mathbf{5}$ & $\mathbf{3 e} / \mathbf{7}$ & $\mathbf{3 f} / \mathbf{9}$ \\
$(S)$-1-(1-naphthyl)ethylamine & $\mathbf{3 g} / \mathbf{1 0}$ & $\mathbf{3 h} / \mathbf{1 2}$ & $\mathbf{3 i} / \mathbf{1 4}$ \\
(R)-1-(1-naphthyl)ethylamine & $\mathbf{3 j} / \mathbf{1 1}$ & $\mathbf{3 k} / \mathbf{1 3}$ & $\mathbf{3 l} / \mathbf{1 5}$
\end{tabular}

Scheme 1. Synthesis of azetidinones 4-15. i) MWI, $800 \mathrm{~W}, 3-4$ min. ii) $\mathrm{PhCH}_{2} \mathrm{CH}_{2} \mathrm{COCl}, \mathrm{Et}_{3} \mathrm{~N}$, toluene, reflux, 24 h. iii) $\mathrm{PhCH}_{2} \mathrm{CH}_{2} \mathrm{COCl}, \mathrm{Et}_{3} \mathrm{~N}$, xylene, reflux, 3 h.

The enantiomeric 1-(2-naphthyl)ethylamines, analogues of phenylethylamines, were studied as a first series. The products were obtained diastereoselectively, just as in the reaction with phenylethylamine, ${ }^{13 \mathrm{c}}$ as pairs of isomers with the desired trans $-\mathrm{C}_{3}, \mathrm{C}_{4}$-configuration, i.e., $(3 R, 4 S)$ and $(3 S, 4 R) ; J_{34}$-constants of $2.0-2.1 \mathrm{~Hz}$ were measured in the proton NMR spectra. The isomeric ratios were determined from the relative integral intensities of the $\mathrm{CH}-4, \mathrm{CH}-\mathrm{Me}$, and $\mathrm{CH}_{3}$ proton NMR resonances, which are in areas free of other signals. The individual diastereoisomers were isolated by high performance flash chromatography (HPFC) on silica gel. As they possess very close or equal $\mathrm{R}_{\mathrm{f}}$-values, a mobile phase with a gradient of polarity was used and pure isomers or enriched fractions were obtained, respectively. The absolute configurations of the products were assumed to be identical with those of the corresponding $N$-(1-phenylethyl) derivatives, ${ }^{13 \mathrm{c}}$ as the NMR data of both groups of compounds display analogous behaviour of the azetidinone skeleton nuclei.

As seen from Table 1, comparable results were obtained with 1-(2-naphthyl)ethylamines and 1-phenylethylamines (the latter given in parentheses). Effective ring construction was achieved with low to moderate asymmetric induction depending on the aldehyde aromatic substituents. The reaction in refluxing xylene afforded the products with similar yields and selectivities (pathway B $v s$ pathway A) but much faster than in toluene ( $3 \mathrm{~h} v s 24 \mathrm{~h}$ ). In all cases, a majority of the less polar isomer was observed. As with phenylethylamine auxiliaries, the best selectivity was achieved with 2,4,6-trimethoxybenzaldehyde (entries 9-12), while 3,4-dimethoxy (entries 58) and 4-dimethylamino (entries 1-4) substituted azetidinones 4-7 were obtained in similar yields and slightly lower selectivities. 
Table 1. Synthesis of $N$-(1-(2-naphthyl)ethyl)-azetidin-2-ones 4-9

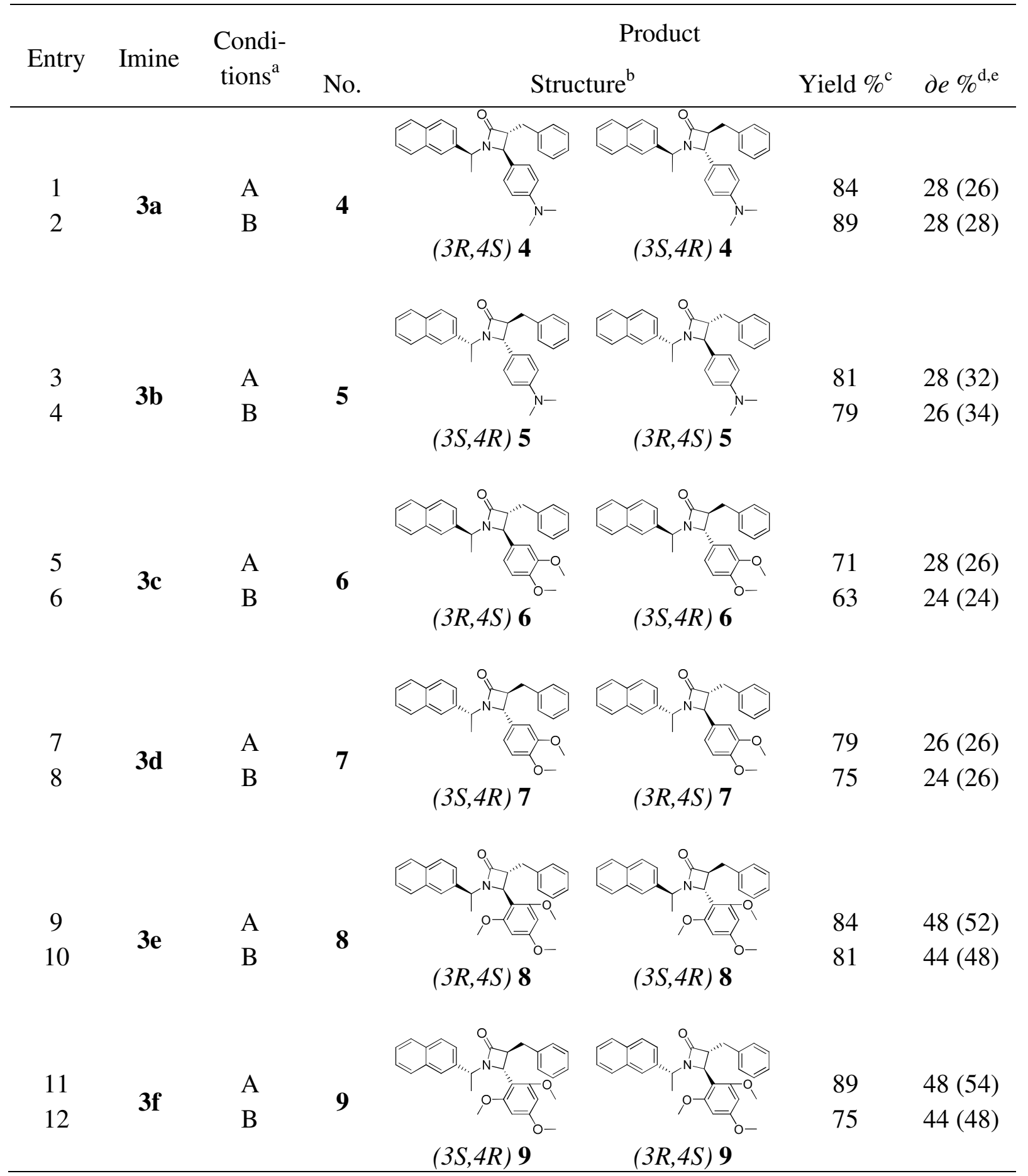

${ }^{\mathrm{a}} \mathrm{A}: 110{ }^{\circ} \mathrm{C}, 24 \mathrm{~h} . \mathrm{B}: 140{ }^{\circ} \mathrm{C}, 2-3 \mathrm{~h}$.

${ }^{\mathrm{b}}$ The major diastereoisomer is depicted first.

${ }^{\mathrm{c}}$ After HPFC purification on silica gel.

${ }^{\mathrm{d}}$ Determined by ${ }^{1} \mathrm{H}$ NMR spectra of the crude mixtures.

${ }^{\mathrm{e}}$ The data for the corresponding $N$-(1-phenylethyl)-derivatives ${ }^{13 \mathrm{c}}$ are given in parenthesis. 
Table 2. Synthesis of $N$-(1-(1-naphthyl)ethyl)-azetidin-2-ones 10-15

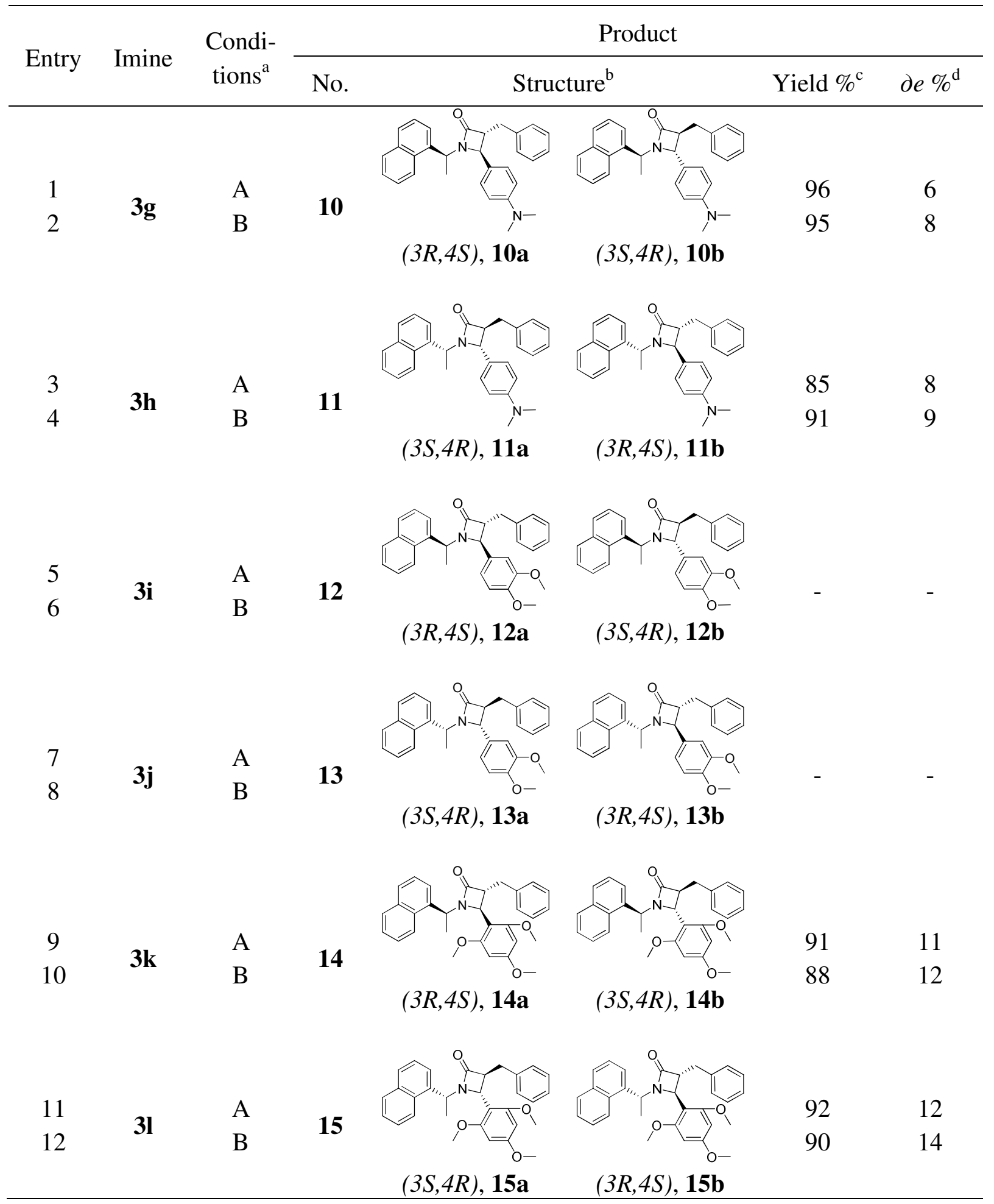

${ }^{\mathrm{a}} \mathrm{A}: 110{ }^{\circ} \mathrm{C}, 24 \mathrm{~h} . \mathrm{B}: 140{ }^{\circ} \mathrm{C}, 3 \mathrm{~h} .{ }^{\mathrm{b}}$ The major diastereoisomer is depicte first.

${ }^{\mathrm{c}}$ After HPFC purification on silica gel. ${ }^{\mathrm{d}}$ Determined by ${ }^{1} \mathrm{H}$ NMR spectra of the crude mixtures. 
As a second series, the effectiveness of the enantioisomeric 1-(1-naphthyl)ethylamines, analogues of phenylethylamine with increased steric hindrance, were examined. Generally, excellent conversions were achieved, up to $96 \%$ of isolated products, but with dramatic loss of selectivity, with a miximum of $14 \%$ de (Table 2). In the case of 3,4-dimethoxyphenyl compounds 12 and 13 (entries 5-8), decomposition took place during chromatography, despite altering the mobile phase, and the products were not isolated. These results show that 1-(1naphthyl)ethylamines are not appropriate chiral auxiliaries in the transformation studied.

The in vitro antimicrobial activities of azetidinones 4-15 as well as all previously obtained $N$ (1-phenylethyl) and $N$-methylpinane compounds ${ }^{13 \mathrm{c}}$ were evaluated qualitatively against 10 bacterial and 3 fungal species. The antibacterial activities were examined against the Gram (+) strains Bacillus subtilis ATCC 6633, Bacillus idosus B241, Bacillus megaterium NRRL 1353895, Bacillus mycoides DSMZ 274, Bacillus cereus ATCC 11778, Acinetobacter johnstonii ATCC 17909, Staphylococcus aureus NRRL B 313, Sarcina lutea ATCC 9341, and Micrococcus luteus ATCC 9631, and the Gram (-) strain Escherichia coli ATCC 8739. The antifungal activities were evaluated against the yeast strains Candida tropicalis ATCC20336, Saccharomyces cerevisiae ATCC 9763, and the fungal strain Penicillium chrysogenum CECT 2802. The products were screened in concentrations $50 \mathrm{mg} / \mathrm{ml}$ in DMSO and the antimicrobial activity was estimated by measuring the diameter of growth of inhibitory zones $(\mathrm{mm})$ in the agar layer. Unfortunately, all compounds tested showed insignificant activities; comparable zones of inhibition of maximum 10-12 mm being measured in all cases.

\section{Conclusions}

In summary, ten pairs of $N$-1-naphthylethyl- $\beta$-lactams with trans- $\mathrm{C}_{3}, \mathrm{C}_{4}$-configuration were obtained via Staudinger cycloaddition with low to moderate diastereoselectivity induced by a chiral amine component of the imine. It was found that 1-(2-naphthyl)ethylamine possess comparable effectiveness as a chiral auxiliary as 1-phenylethylamine, while 1-(1naphthyl)ethylamine led to dramatical loss of selectivity. The azetidinones obtained as well as the previously prepared $N$-(1-phenylethyl) and $N$-methylpinane compounds were screened against 10 bacteria and 3 fungi but significant antimicrobial activities were not observed.

\section{Experimental Section}

General. All reagents were purchased from Aldrich, Merck and Fluka and were used without any further purification. Toluene and xylene were dried over sodium wire. The microwave irradiated reactions (MWI) were performed in domestic household oven Panasonic NN-S255W. Fluka silica gel/TLC-cards 60778 with fluorescent indicator $254 \mathrm{~nm}$ were used for TLC chromatography and Rf-values determination. The high performance flash chromatography 
(HPFC) purifications were carried out on a Biotage Horizon TM system (Charlottesville, Virginia, USA) on silica gel. The melting points were determined in capillary tubes on SRS MPA100 OptiMelt (Sunnyvale, CA, USA) automated melting point system. The NMR spectra were recorded on a Bruker Avance DRX 250 (intermediate imines) and Bruker Avance II+ 600 (final azetidinones) spectrometers (Rheinstetten, Germany) in deuterochloroform; the chemical shifts were quoted in ppm in $\delta$ values against tetramethylsilane (TMS) as an internal standard and the coupling constants were calculated in Hz. The chemical shifts are given with different decimals according to the accuracy (fid resolution) of the corresponding experiments. The assignments of signals were confirmed by 2D techniques (COSY, NOESY, HSQC, HMBC). For simplicity, the aromatic groups are designated as: Naphth - naphthyl; Ph - substituent at C-3, Ar - substituent at C-4. The low resolution mass spectra were taken on a HP 5973 Mass Selective Detector, the high resolution mass spectra on a DFS High Resolution Magnetic Sector MS, Thermo Scientific.

\section{Imines (3a-31). Typical procedure}

A mixture of a chiral amine $\mathbf{1}(1 \mathrm{mmol})$ and an aromatic aldehyde $\mathbf{2}(1 \mathrm{mmol})$ was irradiated in a domestic household microwave oven in an open vessel with a power of $800 \mathrm{~W}$ for 3-4 min. The effectively pure crude products (NMR) were used in the next step without purification.

(S)- $N$-(4-Dimethylaminobenzylidene)-1-(2-naphthyl)ethylamine (3a). 3 min, $98 \%$ yield. (R)- $\boldsymbol{N}$-(4-Dimethylaminobenzylidene)-1-(2-naphthyl)ethylamine (3b). $3 \mathrm{~min}, 99 \%$ yield.

3a and 3b. ${ }^{1} \mathrm{H}$ NMR 1.66 (d, 3H, $J$ 6.6, $\left.\mathrm{CH}_{3}-\mathrm{CH}\right), 3.00$ (s, 6H, $\left.2 \times \mathrm{CH}_{3}-\mathrm{N}\right), 4.64$ (q, $1 \mathrm{H}, J$ 6.6, 13.3, $\mathrm{CH}-\mathrm{CH}_{3}$ ), 6.69 (dt, 2H, J 1.9, 8.9, CH-3 and $\mathrm{CH}-5 \mathrm{Ar}$ ), 7.42 (m, 2H, CH Naphth), 7.57 (dd, 1H, J 1.7, 8.5, CH Naphth), 7.68 (dt, 2H, J 1.9, 8.9, CH-2 and CH-6 Ar), 7.82 (m, 4H, CH Naphth), 8.29 (s, 1H, CH=N). ${ }^{13} \mathrm{C}$ NMR $24.8\left(\mathrm{CH}_{3}-\mathrm{CH}\right), 40.2\left(\mathrm{CH}_{3}-\mathrm{N}\right), 69.5\left(\mathrm{CH}-\mathrm{CH}_{3}\right), 111.6$ $(\mathrm{CH}-3$ and $\mathrm{CH}-5 \mathrm{Ar}), 124.7$ ( $\left.C_{\text {quat }}-1 \mathrm{Ar}\right), 124.8$ ( $\mathrm{CH}$ Naphth), 125.3 ( $C \mathrm{H}$ Naphth), $125.6(\mathrm{CH}$ Naphth), 125.8 ( $C H$ Naphth), 127.6 ( $C H$ Naphth), 127.9 (2x CH Naphth), 129.7 (CH-2 and $C H-6$ $\mathrm{Ar}), 132.6$ ( $C_{\text {quat }}$ Naphth), 133.6 ( $C_{\text {quat }}$ Naphth), 143.3 ( $C_{\text {quat }}$ Naphth), 152.1 ( $\left.C_{\text {quat }}-4 \mathrm{Ar}\right), 159.7$ $(\mathrm{CH}=\mathrm{N}), \mathrm{MS} m / z, 302$.

(S)- $N$-(3,4-Dimethoxybenzylidene)-1-(2-naphthyl)ethylamine (3c). 4 min, 99\% yield.

(R)- $\mathbf{N}$-(3,4-Dimethoxybenzylidene)-1-(2-naphthyl)ethylamine (3d). $4 \mathrm{~min}, 99 \%$ yield.

3c and 3d. ${ }^{1} \mathrm{H}$ NMR $1.58\left(\mathrm{~d}, 3 \mathrm{H}, J\right.$ 6.7, $\left.\mathrm{CH}_{3}-\mathrm{CH}\right), 3.91$ (s, 3H, OCH$), 3.96$ (s, 3H, OCH $), 4.69$ (q, 1H, J 6.7, 13.2, $\mathrm{CH}-\mathrm{CH}_{3}$ ), 6.88 (d, 1H, J 8.2, CH-5 Ar), 7.20 (dd, 1H, J 1.9, 8.2, CH-6 Ar), 7.44 (dd, 1H, J 1.9, 9.4, CH Naphth), 7.44 (s, 1H, CH-1 Naphth), 7.50 (d, 1H, J 1.9, CH-2 Ar), 7.58 (dd, 1H, J 1.6, 8.5, CH Naphth), 7.82 (m, 4H, CH Naphth), 8.33 (s, 1H, CH=N). ${ }^{13} \mathrm{C}$ NMR $24.6\left(\mathrm{CH}_{3}-\mathrm{CH}\right), 55.9\left(\mathrm{OCH}_{3}\right), 56.0\left(\mathrm{OCH}_{3}\right), 69.5\left(\mathrm{CH}-\mathrm{CH}_{3}\right), 109.2(\mathrm{CH}-2 \mathrm{Ar}), 110.5(\mathrm{CH}-5 \mathrm{Ar})$, 123.0 ( $\mathrm{CH}-6 \mathrm{Ar}), 124.9$ ( $\mathrm{CH}$ Naphth), 125.4 ( $\mathrm{CH}$ Naphth), 125.5 ( $C \mathrm{H}$ Naphth), 125.9 ( $C \mathrm{H}$ Naphth), 127.6 ( $C H$ Naphth), 127.9 ( $C H$ Naphth), 128.0 ( $C H$ Naphth), 129.8 ( $\left.C_{\text {quat }}-1 \mathrm{Ar}\right), 132.6$ $\left(C_{\text {quat }}\right.$ Naphth), 133.5 ( $C_{\text {quat }}$ Naphth), 142.7 ( $C_{\text {quat }}$ Naphth), 149.3 ( $C_{\text {quat }}-3$ or $\left.C_{\text {quat }}-4 \mathrm{Ar}\right), 151.3$ $\left(C_{\text {quat }}-3\right.$ or $\left.C_{\text {quat }}-4 \mathrm{Ar}\right), 159.2(\mathrm{CH}=\mathrm{N}), \mathrm{MS} m / z 319$.

(S)-N-(2,4,6-Trimethoxybenzylidene)-1-(2-naphthyl)ethylamine (3e). $3 \mathrm{~min}, 99 \%$ yield. 
(R)- $\mathbf{N}$-(2,4,6-Trimethoxybenzylidene)-1-(2-naphthyl)ethylamine (3f). $3 \mathrm{~min}, 98 \%$ yield.

3e and 3f. ${ }^{1} \mathrm{H}$ NMR $1.68\left(\mathrm{~d}, 3 \mathrm{H}, J\right.$ 6.6, $\left.\mathrm{CH}_{3}-\mathrm{CH}\right), 3.82$ (s, 3H, OCH $\mathrm{O}_{3}, 3.84$ (s, 6H, 2 x $\left.\mathrm{OCH}_{3}\right)$, 4.63 (q, 1H, J 6.6, 13.2, $\mathrm{CH}-\mathrm{CH}_{3}$ ), 6.13 (s, 2H, CH-3 and $\mathrm{CH}-5 \mathrm{Ar}$ ), 7.42 (m, 2H, CH Naphth), 7.61 (dd, 1H, J 1.7, 8.6, CH Naphth), 7.81 (m, 3H, CH Naphth), 7.95 (d, 1H, J 0.9, CH-1 Naphth), 8.61 (s, $1 \mathrm{H}, \mathrm{CH}=\mathrm{N}) .{ }^{13} \mathrm{C} \mathrm{NMR} 25.2\left(\mathrm{CH}_{3}-\mathrm{CH}\right), 55.3\left(\mathrm{OCH}_{3}\right), 56.0\left(2 \times \mathrm{OCH}_{3}\right), 71.0$ $\left(\mathrm{CH}-\mathrm{CH}_{3}\right), 90.8(\mathrm{CH}-3$ and $\mathrm{CH}-5 \mathrm{Ar}), 107.9\left(C_{\text {quat }}-1 \mathrm{Ar}\right), 124.9(\mathrm{CH}$ Naphth$), 125.2(\mathrm{CH}$ Naphth), 125.5 ( $C H$ Naphth), 125.6 ( $C H$ Naphth), 127.5 ( $C H$ Naphth), 127.6 ( $C H$ Naphth), 127.9 ( $C H$ Naphth), 132.4 ( $C_{\text {quat }}$ Naphth), 133.6 ( $C_{\text {quat }}$ Naphth), 143.8 ( $C_{\text {quat }}$ Naphth), 154.8 $(C \mathrm{H}=\mathrm{N}), 160.7\left(C_{\text {quat }}-2\right.$ and $\left.C_{\text {quat }}-6 \mathrm{Ar}\right), 162.4\left(C_{\text {quat }}-4 \mathrm{Ar}\right), \mathrm{MS} \mathrm{m} / \mathrm{z} 349$.

(S)- $N$-(4-Dimethylaminobenzylidene)-1-(1-naphthyl)ethylamine (3g). $4 \mathrm{~min}, 99 \%$ yield.

(R)- $\boldsymbol{N}$-(4-Dimethylaminobenzylidene)-1-(1-naphthyl)ethylamine (3h). $4 \mathrm{~min}, 98 \%$ yield.

3g and 3h. ${ }^{1} \mathrm{H}$ NMR 1.72 (d, 3H, $J$ 6.6, $\left.\mathrm{CH}_{3}-\mathrm{CH}\right), 2.98$ (s, 6H, 2 x $\left.\mathrm{CH}_{3}-\mathrm{N}\right), 5.28$ (q, 1H, $J$ 6.6, 13.2, $\left.\mathrm{CH}-\mathrm{CH}_{3}\right), 6.68$ (d, 2H, J 8.9, CH-3 and $\mathrm{CH}-5 \mathrm{Ar}$ ), 7.47 (m, 3H, CH Naphth), 7.67 (d, 2H, J 8.9, CH-2 and CH-6 Ar), 7.73 (d, 1H, J 8.2, CH Naphth), 7.84 (m, 2H, CH Naphth), 8.25 (d, 1H, $J$ 8.3, CH Naphth), 8.29 (s, $1 \mathrm{H}, \mathrm{CH}=\mathrm{N}) .{ }^{13} \mathrm{C} \mathrm{NMR} 24.7\left(\mathrm{CH}_{3}-\mathrm{CH}\right), 40.2\left(\mathrm{CH}_{3}-\mathrm{N}\right), 65.3(\mathrm{CH}-$ $\left.\mathrm{CH}_{3}\right), 111.6$ ( $\mathrm{CH}-3$ and $\left.\mathrm{CH}-5 \mathrm{Ar}\right), 123.8$ ( $\mathrm{CH}$ Naphth), 124.0 ( $\mathrm{CH}$ Naphth), 124.8 ( $\left.C_{\text {quat }}-1 \mathrm{Ar}\right)$, 125.2 ( $C H$ Naphth), 125.6 ( $C H$ Naphth), 125.7 ( $C H$ Naphth), 127.1 ( $C H$ Naphth), 128.8 (CH Naphth), 129.6 ( $C H-2$ and $C H-6$ Ar), 130.8 ( $C_{\text {quat }}$ Naphth), 134.0 ( $C_{\text {quat }}$ Naphth), 141.9 ( $C_{\text {quat }}$ Naphth), 152.1 ( $\left.C_{\text {quat }}-4 \mathrm{Ar}\right), 159.5(\mathrm{CH}=\mathrm{N}), \mathrm{MS} m / z, 302$.

(S)- $N$-(3,4-Dimethoxybenzylidene)-1-(1-naphthyl)ethylamine (3i). $4 \mathrm{~min}, 99 \%$ yield.

(R)-N-(3,4-Dimethoxybenzylidene)-1-(1-naphthyl)ethylamine (3j). $4 \mathrm{~min}, 99 \%$ yield.

3i and 3j. ${ }^{1} \mathrm{H}$ NMR 1.75 (d, 3H, $J$ 6.7, $\left.\mathrm{CH}_{3}-\mathrm{CH}\right), 3.92$ (s, 3H, $\left.\mathrm{OCH}_{3}\right), 3.96$ (s, 3H, OCH $), 5.36$ (q, 1H, J 6.7, 13.2, $\mathrm{CH}-\mathrm{CH}_{3}$ ), 6.88 (d, 1H, J 8.2, CH-5 Ar), 7.20 (dd, 1H, J 1.9, 8.2, CH-6 Ar), 7.52 (m, 4H, CH-2 Ar and $3 \mathrm{CH}$ Naphth), 7.87 (m, 3H, CH Naphth), 8.26 (dd, 1H, J 1.2, 8.2, CH Naphth), $8.34(\mathrm{~s}, 1 \mathrm{H}, \mathrm{CH}=\mathrm{N}) .{ }^{13} \mathrm{C} \mathrm{NMR} 24.4\left(\mathrm{CH}_{3}-\mathrm{CH}\right), 56.0\left(\mathrm{OCH}_{3}\right), 56.1\left(\mathrm{OCH}_{3}\right), 65.2(\mathrm{CH}-$ $\left.\mathrm{CH}_{3}\right), 109.2$ ( $\left.\mathrm{CH}-2 \mathrm{Ar}\right), 110.4$ ( $\left.\mathrm{CH}-5 \mathrm{Ar}\right), 122.9$ ( $\mathrm{CH}$ Naphth), 123.6 ( $\mathrm{CH}$ Naphth), 124.0 ( $\mathrm{CH}$ Naphth), 125.2 ( $\mathrm{CH}$ Naphth), 125.6 ( $\mathrm{CH}$ Naphth), 125.9 ( $\mathrm{CH}$ Naphth),127.2 (CH-6 Ar), 128.8 (CH Naphth), 129.8 ( $\left.C_{\text {quat }}-1 \mathrm{Ar}\right), 130.7$ ( $C_{\text {quat }}$ Naphth), 133.9 ( $C_{\text {quat }}$ Naphth), 141.3 ( $C_{\text {quat }}$ Naphth), 149.2 ( $C_{\text {quat }}-3$ or $\left.C_{\text {quat }}-4 \mathrm{Ar}\right), 151.3$ ( $C_{\text {quat }}-3$ or $\left.C_{\text {quat }}-4 \mathrm{Ar}\right), 159.0(C \mathrm{H}=\mathrm{N}), \mathrm{MS} m / z 319$.

(S)- $N$-(2,4,6-Trimethoxybenzylidene)-1-(1-naphthyl)ethylamine (3k). 4 min, $98 \%$ yield. (R)-N-(2,4,6-Trimethoxybenzylidene)-1-(1-naphthyl)ethylamine (3I). $4 \mathrm{~min}, 99 \%$ yield.

3k and 31. ${ }^{1} \mathrm{H}$ NMR 1.75 (d, 3H, J 6.6, $\left.\mathrm{CH}_{3}-\mathrm{CH}\right), 3.82$ (s, 3H, $\left.\mathrm{OCH}_{3}\right), 3.83$ (s, 6H, 2 x $\mathrm{OCH}_{3}$ ), 5.22 (q, 1H, J 6.6, 13.2, $\left.\mathrm{CH}-\mathrm{CH}_{3}\right), 6.11$ (s, 2H, CH-3 and $\mathrm{CH}-5 \mathrm{Ar}$ ), 7.49 (m, 3H, CH Naphth), 7.73 (d, 1H, J 8.2, CH Naphth), 7.86 (m, 2H, CH Naphth), 8.35 (dd, 1H, J 1.0, 8.3, CH Naphth), $8.65(\mathrm{~s}, 1 \mathrm{H}, \mathrm{CH}=\mathrm{N}) .{ }^{13} \mathrm{C}$ NMR $25.0\left(\mathrm{CH}_{3}-\mathrm{CH}\right), 55.5\left(\mathrm{OCH}_{3}\right), 56.2\left(2 \times \mathrm{OCH}_{3}\right), 67.9\left(\mathrm{CH}-\mathrm{CH}_{3}\right)$, 91.0 ( $\mathrm{CH}-3$ and $\mathrm{CH}-5 \mathrm{Ar}), 121.5$ ( $\left.C_{\text {quat }}-1 \mathrm{Ar}\right), 124.4$ (2xCH Naphth), 125.2 (CH Naphth), 125.5 (CH Naphth), 125.8 ( $C H$ Naphth), 127.1 ( $C H$ Naphth), 128.9 ( $C H$ Naphth), 130.8 ( $C_{\text {quat }}$ Naphth), 134.1 ( $C_{\text {quat }}$ Naphth), $142.6\left(C_{\text {quat }}\right.$ Naphth), $154.9(C \mathrm{H}=\mathrm{N}), 161.0\left(C_{\text {quat }}-2\right.$ and $\left.C_{\text {quat }}-6 \mathrm{Ar}\right), 162.6$ ( $\left.C_{\text {quat }}-4 \mathrm{Ar}\right), \mathrm{MS} \mathrm{m} / \mathrm{z} 349$. 


\section{Azetidin-2-ones (4-15). Typical procedure}

To a refluxing solution of imine 3 (1 mmol) and $\mathrm{Et}_{3} \mathrm{~N}(1.5 \mathrm{mmol})$ in toluene or xylene (15 ml) 3phenylpropionyl chloride $(1 \mathrm{mmol})$ was added and the mixture was refluxed for $24 \mathrm{~h}$ (in toluene) or for $3 \mathrm{~h}$ (in xylene). The products were partitioned between water and toluene/xylene. The organic phase was washed with brine, dried over $\mathrm{Na}_{2} \mathrm{SO}_{4}$, evaporated to dryness, and purified by HPFC on silica gel. The mobile phase used was: a) $\mathrm{CH}_{2} \mathrm{Cl}_{2}$ :acetone 100:1 for diastereoisomers with $\mathrm{R}_{\mathrm{f}}$-difference at least 0.1 ; b) with a gradient of polarity from $\mathrm{CH}_{2} \mathrm{Cl}_{2}$ to $\mathrm{CH}_{2} \mathrm{Cl}_{2}$ :acetone 100:1 for compounds possessing very close $\mathrm{R}_{\mathrm{f}}$-values. The yields and selectivities are summarized on Table 1.

3-Benzyl-4-(4-(dimethylamino)phenyl)-1-((S)-1-(2-napthyl)ethyl)azetidin-2-one (4) and 3benzyl-4-(4-(dimethylamino)phenyl)-1-((R)-1-(2-napthyl)ethyl)azetidin-2-one (5). $\mathrm{MS} \mathrm{m} / \mathrm{z}$ 434. HRMS (EI): calcd for $\mathrm{C}_{30} \mathrm{H}_{30} \mathrm{~N}_{2} \mathrm{O}\left(\mathrm{M}^{+}\right)$: 434.2358, found: 434.2337.

(3R,4S)-4 and (3S,4R)-5. $\mathrm{R}_{\mathrm{f}} 0.29\left(\mathrm{CH}_{2} \mathrm{Cl}_{2}\right.$ :acetone 100:1). ${ }^{1} \mathrm{H}$ NMR $1.788\left(\mathrm{~d}, 3 \mathrm{H}, \mathrm{J} 7.2, \mathrm{CH}_{3^{-}}\right.$ $\mathrm{CH}), 2.903\left(\mathrm{~s}, 6 \mathrm{H}, 2 \times \mathrm{CH}_{3}-\mathrm{N}\right.$ ), 3.022 (A part of $\left.\mathrm{ABX}, 1 \mathrm{H}, J_{\mathrm{AX}} 8.0, J_{\mathrm{AB}} 14.7,1 / 2 \mathrm{CH}_{2}\right), 3.100(\mathrm{~B}$ part of $\mathrm{ABX}, 1 \mathrm{H}, J_{\mathrm{BX}} 5.2, J_{\mathrm{AB}} 14.7,1 / 2 \mathrm{CH}_{2}$ ), 3.291 (ddd, $1 \mathrm{H}, J 2.0,5.2,8.0, \mathrm{CH}-3$ ), 3.951 (d, $1 \mathrm{H}$, $J$ 2.0, $\mathrm{CH}-4$ ), 4.313 (q, 1H, J 7.2, 14.3, $\mathrm{CH}_{-} \mathrm{CH}_{3}$ ), 6.565 (dt, 2H, J 2.0, 8.7, CH-3 and $\mathrm{CH}-5 \mathrm{Ar}$ ), 6.924 (dt, 2H, J 2.0, 8.7, $\mathrm{CH}-2$ and $\mathrm{CH}-6 \mathrm{Ar}$ ), 7.183 (m, 4H, $\mathrm{CH}-3 \mathrm{Naphth}$ and $\mathrm{CH}-2$, $\mathrm{CH}-4$ and $\mathrm{CH}-6 \mathrm{Ph}$ ), 7.229 (t, 2H, J 7.6, CH-3 and $\mathrm{CH}-5 \mathrm{Ph}$ ), 7.366 (s, 1H, CH-1 Naphth), 7.439 (m, 2H, CH-7 and CH-8 Naphth), 7.679 (dd, 1H, J 2.8, 9.4, CH-9 Naphth), 7.693 (d, 1H, J 8.5, CH-4 Naphth), 7.787 (dd, 1H, J 2.8, 9.3, CH-6 Naphth). ${ }^{13} \mathrm{C} \mathrm{NMR} 20.35\left(\mathrm{CH}_{3}-\mathrm{CH}\right), 33.96\left(\mathrm{CH}_{2}\right)$, $40.48\left(\mathrm{CH}_{3}-\mathrm{N}\right), 54.53\left(\mathrm{CH}-\mathrm{CH}_{3}\right), 58.95(\mathrm{CH}-4), 59.59(\mathrm{CH}-3), 112.40(\mathrm{CH}-3$ and $\mathrm{CH}-5 \mathrm{Ar})$, 124.84 ( $\left.C_{\text {quat }}-1 \mathrm{Ar}\right), 124.87$ (CH-3 Naphth), 125.34 (CH-1 Naphth), 125.79 (CH-8 Naphth), 125.99 ( $\mathrm{CH}-7$ Naphth), 126.44 ( $\mathrm{CH}-4 \mathrm{Ph}), 127.59$ (CH-6 Naphth), 127.71 ( $\mathrm{CH}-2$ and $\mathrm{CH}-6 \mathrm{Ar})$,

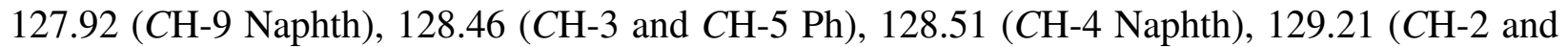
CH-6 Ph), 132.70 ( $C_{\text {quat }}-5$ Naphth), 133.18 ( $C_{\text {quat }}-10$ Naphth), 138.06 ( $\left.C_{\text {quat }}-1 \mathrm{Ph}\right), 139.18\left(C_{\text {quat }^{-}}\right.$ 2 Naphth), 150.40 ( $\left.C_{\text {quat }}-4 \mathrm{Ar}\right), 170.07(C=\mathrm{O})$.

(3S,4R)-4 and (3R,4S)-5. $\mathrm{R}_{\mathrm{f}} 0.24\left(\mathrm{CH}_{2} \mathrm{Cl}_{2}\right.$ :acetone 100:1). ${ }^{1} \mathrm{H}$ NMR 1.333 (d, 3H, J 7.2, $\mathrm{CH}_{3^{-}}$ $\mathrm{CH}), 2.931\left(\mathrm{~s}, 6 \mathrm{H}, 2 \times \mathrm{CH}_{3} \mathrm{-N}\right), 2.936$ (A part of $\left.\mathrm{ABX}, 1 \mathrm{H}, J_{\mathrm{AX}} 7.6, J_{\mathrm{AB}} 14.8,1 / 2 \mathrm{CH}_{2}\right), 3.006(\mathrm{~B}$ part of $\left.\mathrm{ABX}, 1 \mathrm{H}, J_{\mathrm{BX}} 5.4, J_{\mathrm{AB}} 14.8,1 / 2 \mathrm{CH}_{2}\right), 3.313$ (ddd, $1 \mathrm{H}, J$ 2.1, 5.4, 7.6, CH-3), $3.896(\mathrm{~d}, 1 \mathrm{H}$, $J$ 2.1, CH-4), 5.099 (q, 1H, J 7.2, 14.2, $\mathrm{CH}_{-} \mathrm{CH}_{3}$ ), 6.606 (dt, 2H, J 1.9, 8.7, CH-3 and $\mathrm{CH}-5 \mathrm{Ar}$ ), 7.005 (dt, 2H, J 1.9, 8.7, CH-2 and $\mathrm{CH}-6 \mathrm{Ar}$ ), 7.108 (m, 4H, CH-3 Naphth and $\mathrm{CH}-2$, $\mathrm{CH}-4$ and $\mathrm{CH}-6 \mathrm{Ph}), 7.148$ (t, 2H, J 7.6, CH-3 and $\mathrm{CH}-5 \mathrm{Ph}), 7.467$ (m, 2H, CH-7 and CH-8 Naphth), 7.487 (s, 1H, CH-1 Naphth), 7.704 (d, 1H, J 8.5, CH-4 Naphth), 7.735 (dd, 1H, J 2.6, 9.4, CH-9 Naphth), 7.810 (dd, 1H, J 2.6, 9.3, CH-6 Naphth). ${ }^{13} \mathrm{C} \mathrm{NMR} 18.72\left(\mathrm{CH}_{3}-\mathrm{CH}\right), 33.73\left(\mathrm{CH}_{2}\right)$, $40.49\left(\mathrm{CH}_{3}-\mathrm{N}\right), 51.52\left(\mathrm{CH}_{-} \mathrm{CH}_{3}\right), 59.21(\mathrm{CH}-4), 59.52(\mathrm{CH}-3), 112.27(\mathrm{CH}-3$ and $\mathrm{CH}-5 \mathrm{Ar})$, 125.72 (CH-3 Naphth), 125.73 (CH-8 Naphth), 125.98 ( $\mathrm{CH}-7$ Naphth), 126.07 (CH-1 Naphth), 126.15 ( $\left.C_{\text {quat }}-1 \mathrm{Ar}\right), 126.38(\mathrm{CH}-4 \mathrm{Ph}), 127.61$ ( $\left.\mathrm{CH}-6 \mathrm{Naphth}\right), 127.92$ ( $\mathrm{CH}-2$ and $\left.\mathrm{CH}-6 \mathrm{Ar}\right)$, 128.11 (CH-9 Naphth), 128.30 ( $\mathrm{CH}-4$ Naphth), 128.39 (CH-3 and $\mathrm{CH}-5 \mathrm{Ph}), 129.14$ (CH-2 and CH-6 Ph), 132.71 ( $C_{\text {quat }}-5$ Naphth), 132.73 ( $C_{\text {quat }}-10$ Naphth), 137.45 ( $C_{\text {quat }}-2$ Naphth), 137.88 $\left(C_{\text {quat }}-1 \mathrm{Ph}\right), 150.46$ ( $\left.C_{\text {quat }}-4 \mathrm{Ar}\right), 170.17(C=\mathrm{O})$. 
3-Benzyl-4-(3,4-dimethoxyphenyl)-1-((S)-1-(2-napthyl)ethyl)azetidin-2-one (6) and 3-benzyl -4-(3,4-dimethoxyphenyl)-1-((R)-1-(2-napthyl)ethyl)azetidin-2-one (7). MS $\mathrm{m} / \mathrm{z}$ 451. HRMS (EI): calcd for $\mathrm{C}_{30} \mathrm{H}_{29} \mathrm{NO}_{3}\left(\mathrm{M}^{+}\right)$: 451.2147, found: 451.2189 .

(3R,4S)-6 and (3S,4R)-7. $\mathrm{R}_{\mathrm{f}} 0.34\left(\mathrm{CH}_{2} \mathrm{Cl}_{2}\right.$ :acetone 100:1). ${ }^{1} \mathrm{H}$ NMR 1.782 (d, 3H, J 7.2, $\mathrm{CH}_{3^{-}}$ $\mathrm{CH}$ ), 3.006 (A part of $\mathrm{ABX}, 1 \mathrm{H}, J_{\mathrm{AX}} 8.5, \mathrm{~J}_{\mathrm{AB}} 14.6,1 / 2 \mathrm{CH}_{2}$ ), 3.149 (B part of ABX, 1H, $J_{\mathrm{BX}} 5.1$, $J_{\mathrm{AB}} 14.6,1 / 2 \mathrm{CH}_{2}$ ), 3.268 (ddd, $1 \mathrm{H}, J 2.1,5.1,8.5, \mathrm{CH}-3$ ), 3.541 (s, 3H, $\mathrm{OCH}_{3}-3$ ), 3.808 (s, 3H, $\left.\mathrm{OCH}_{3}-4\right), 4.014$ (d, 1H, J 2.1, CH-4), 4.472 (q, 1H, J 7.2, 14.3, $\left.\mathrm{CH}-\mathrm{CH}_{3}\right), 6.328$ (d, 1H, J 1.9, CH-2 Ar), 6.598 (dd, 1H, J 1.9, 8.2, CH-6 Ar), 6.685 (d, 1H, J 8.2, CH-5 Ar), 7.194 (m, 4H, CH3 Naphth and $\mathrm{CH}-2, \mathrm{CH}-4$ and $\mathrm{CH}-6 \mathrm{Ph}$ ), 7.249 (t, 2H, J 7.5, CH-3 and $\mathrm{CH}-5 \mathrm{Ph}$ ), 7.411 (s, 1H, CH-1 Naphth), 7.445 (m, 2H, CH-7 and CH-8 Naphth), 7.689 (dd, 1H, J 2.9, 9.6, CH-9 Naphth), 7.704 (d, 1H, J 8.5, CH-4 Naphth), 7.788 (dd, 1H, J 2.6, 9.3, CH-6 Naphth). ${ }^{13} \mathrm{C}$ NMR 19.85 $\left(\mathrm{CH}_{3}-\mathrm{CH}\right), 34.07\left(\mathrm{CH}_{2}\right), 54.36\left(\mathrm{CH}_{-} \mathrm{CH}_{3}\right), 55.66\left(\mathrm{OCH}_{3}-3\right), 55.95\left(\mathrm{OCH}_{3}-4\right), 59.23(\mathrm{CH}-4)$, 60.00 (CH-3), 109.10 (CH-2 Ar), 111.04 ( $\mathrm{CH}-5 \mathrm{Ar}), 118.89$ (CH-6 Ar), 124.93 (CH-3 Naphth), 125.63 ( $\mathrm{CH}-1$ Naphth), 126.07 ( $\mathrm{CH}-8$ Naphth), 126.25 ( $\mathrm{CH}-7$ Naphth), 126.65 ( $\mathrm{CH}-4 \mathrm{Ph})$,

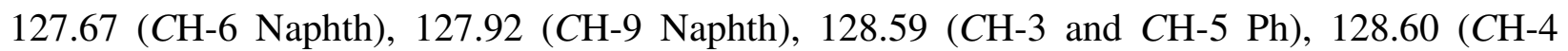

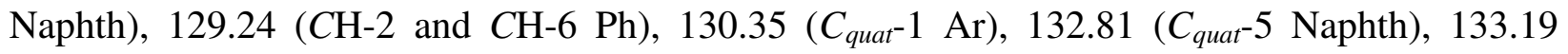
( $C_{\text {quat }}-10$ Naphth), 137.99 ( $\left.C_{\text {quat }}-1 \mathrm{Ph}\right), 138.81$ ( $\left.C_{\text {quat }}-2 \mathrm{Naphth}\right), 148.84$ ( $\left.C_{\text {quat }}-4 \mathrm{Ar}\right), 149.20$ $\left(C_{\text {quat }}-3 \mathrm{Ar}\right), 170.05(C=\mathrm{O})$.

(3S,4R)-6 and (3R,4S)-7. $\mathrm{R}_{\mathrm{f}} 0.29\left(\mathrm{CH}_{2} \mathrm{Cl}_{2}\right.$ :acetone 100:1). ${ }^{1} \mathrm{H}$ NMR ${ }^{1} \mathrm{H}$ NMR 1.421 (d, 3H, $J$ 7.1, $\mathrm{CH}_{3}-\mathrm{CH}$ ), 2.934 (A part of ABX, $1 \mathrm{H}, J_{\mathrm{AX}} 8.3, J_{\mathrm{AB}} 14.6,1 / 2 \mathrm{CH}_{2}$ ), 3.067 (B part of ABX, 1H, $\left.J_{\mathrm{BX}} 5.2, J_{\mathrm{AB}} 14.6,1 / 2 \mathrm{CH}_{2}\right), 3.303\left(\mathrm{ddd}, 1 \mathrm{H}, J\right.$ 2.0, 5.2, 8.3, $\mathrm{CH}-3$ ), 3.615 (s, 3H, $\mathrm{OCH}_{3}-3$ ), 3.848 (s, 3H, $\left.\mathrm{OCH}_{3}-4\right), 3.934$ (d, 1H, J 2.0, CH-4), 5.048 (q, 1H, J 7.1, 14.2, $\left.\mathrm{CH}_{-} \mathrm{CH}_{3}\right), 6.511$ (d, 1H, J 1.8, CH-2 Ar), 6.647 (dd, 1H, J 1.8, 8.2, CH-6 Ar), 6.723 (d, 1H, J 8.2, CH-5 Ar), 7.106 (d, 2H, J 7.2, $\mathrm{CH}-2$ and $\mathrm{CH}-6 \mathrm{Ph}), 7.183$ (m, 4H, CH-3 Naphth and $\mathrm{CH}-3, \mathrm{CH}-4$ and $\mathrm{CH}-5 \mathrm{Ph}), 7.476$ (m, 2H, CH-7 and CH-8 Naphth), 7.512 (s, 1H, CH-1 Naphth), 7.732 (m, 2H, CH-4 and CH-9 Naphth), 7.816 (dd, 1H, J 2.6, 9.2, CH-6 Naphth). ${ }^{13} \mathrm{C}$ NMR $19.02\left(\mathrm{CH}_{3}-\mathrm{CH}\right), 33.85\left(\mathrm{CH}_{2}\right)$, $52.35\left(\mathrm{CH}-\mathrm{CH}_{3}\right), 55.77\left(\mathrm{OCH}_{3}-3\right), 55.96\left(\mathrm{OCH}_{3}-4\right), 59.75(\mathrm{CH}-4), 59.84(\mathrm{CH}-3), 109.28(\mathrm{CH}-2$ Ar), 110.92 ( $C H-5$ Ar), 119.34 (CH-6 Ar), 125.73 (CH-3 Naphth), 125.84 (CH-1 Naphth),

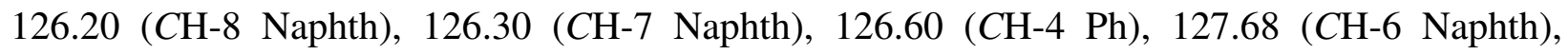

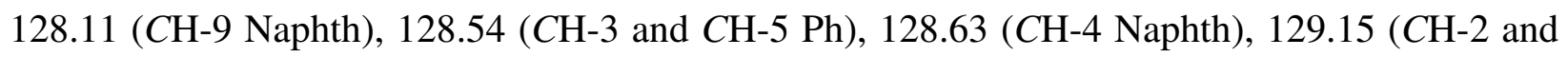
CH-6 Ph), 131.47 ( $\left.C_{\text {quat }}-1 \mathrm{Ar}\right), 132.82$ ( $C_{\text {quat }}-5$ Naphth), 133.24 ( $C_{\text {quat }}-10$ Naphth), 137.46 ( $C_{\text {quat }}{ }^{-}$ 2 Naphth), 137.80 ( $\left.C_{\text {quat }}-1 \mathrm{Ph}\right), 148.96$ ( $\left.C_{\text {quat }}-4 \mathrm{Ar}\right), 149.13$ ( $\left.C_{\text {quat }}-3 \mathrm{Ar}\right), 170.26(C=\mathrm{O})$.

3-Benzyl-4-(2,4,6-trimethoxyphenyl)-1-((S)-1-(2-napthyl)ethyl)azetidin-2-one (8) and 3benzyl-4-(2,4,6-trimethoxyphenyl)-1-((R)-1-(2-napthyl)ethyl)azetidin-2-one (9). MS m/z 481. HRMS (EI): calcd for $\mathrm{C}_{31} \mathrm{H}_{31} \mathrm{NO}_{4}\left(\mathrm{M}^{+}\right)$: 481.2253, found: 481.2274.

(3R,4S)-8 and (3S,4R)-9. $\mathrm{R}_{\mathrm{f}} 0.19\left(\mathrm{CH}_{2} \mathrm{Cl}_{2}\right.$ :acetone 100:1). ${ }^{1} \mathrm{H}$ NMR 1.612 (d, 3H, $J$ 7.1, $\mathrm{CH}_{3^{-}}$ $\mathrm{CH}$ ), 3.022 (A part of $\mathrm{ABX}, 1 \mathrm{H}, J_{\mathrm{AX}} 8.2, J_{\mathrm{AB}} 14.6,1 / 2 \mathrm{CH}_{2}$ ), 3.080 (B part of ABX, 1H, $J_{\mathrm{BX}} 5.1, J$ AB 14.6, 1/2 $\mathrm{CH}_{2}$ ), 3.447 (s, 6H, $\mathrm{OCH}_{3}-2$ and $\mathrm{OCH}_{3}-6$ ), 3.637 (s, 3H, OCH $\left.H_{3}-4\right), 3.686$ (ddd, $1 \mathrm{H}, J$ 2.0, 5.1, 8.2, CH-3), 4.568 (q, 1H, J 7.1, 14.1, $\mathrm{CH}-\mathrm{CH}_{3}$ ), 4.823 (d, 1H, J 2.0, CH-4), 5.779 (s, 2H, CH-3 and $\mathrm{CH}-5 \mathrm{Ar}$ ), 7.113 (dd, $1 \mathrm{H}, J$ 1.7, 8.5, CH-3 Naphth), 7.231 (m, 4H, CH-2, CH-3, 
CH-5 and $\mathrm{CH}-6 \mathrm{Ph}$ ), 7.253 (d, 1H, J 1.7, CH-1 Naphth), 7.288 (dd, 1H, J 7.3, 7.7, CH-4 Ph), 7.392 (m, 2H, CH-7 and CH-8 Naphth), 7.580 (d, 1H, J 8.6, CH-4 Naphth), 7.598 (dd, 1H, J 2.1, 6.8, CH-9 Naphth), 7.725 (dd, $1 \mathrm{H}, J$ 1.9, 9.3, CH-6 Naphth). ${ }^{13} \mathrm{C}$ NMR $18.66\left(\mathrm{CH}_{3}-\mathrm{CH}\right), 34.67$ $\left(\mathrm{CH}_{2}\right), 50.46(\mathrm{CH}-4), 52.67\left(\mathrm{CH}-\mathrm{CH}_{3}\right), 54.68(\mathrm{CH}-3), 55.16\left(\mathrm{OCH}_{3}-4\right), 55.31\left(\mathrm{OCH}_{3}-2\right.$ and $\left.\mathrm{OCH}_{3}-6\right), 90.15$ ( $\mathrm{CH}-3$ and $\left.\mathrm{CH}-5 \mathrm{Ar}\right), 104.25$ ( $\left.C_{\text {quat }}-1 \mathrm{Ar}\right), 125.41$ (CH-1 Naphth), 125.53 (CH-3 Naphth), 125.64 (CH-7 Naphth), 125.67 (CH-8 Naphth), 127.37 (CH-6 Naphth), 127.43 (CH-9

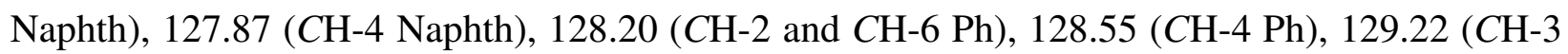
and $C \mathrm{H}-5 \mathrm{Ph}), 132.57$ ( $C_{\text {quat }}-5$ Naphth), 133.05 ( $C_{\text {quat }}-10$ Naphth), 138.28 ( $C_{\text {quat }}-2$ Naphth), $138.92\left(C_{\text {quat }}-1 \mathrm{Ph}\right), 160.05\left(C_{\text {quat }}-2\right.$ and $\left.C_{\text {quat }}-6 \mathrm{Ar}\right), 161.00\left(C_{\text {quat }}-4 \mathrm{Ar}\right), 170.46(C=\mathrm{O})$.

(3S,4R)-8 and (3R,4S)-9. $\mathrm{R}_{\mathrm{f}} 0.16\left(\mathrm{CH}_{2} \mathrm{Cl}_{2}\right.$ :acetone 100:1). ${ }^{1} \mathrm{H}$ NMR $1.336\left(\mathrm{~d}, 3 \mathrm{H}, \mathrm{J} 7.1, \mathrm{CH}_{3^{-}}\right.$ $\mathrm{CH}$ ), 2.924 (A part of $\mathrm{ABX}, 1 \mathrm{H}, J_{\mathrm{AX}} 8.2, J_{\mathrm{AB}} 14.6,1 / 2 \mathrm{CH}_{2}$ ), 2.977 (B part of ABX, 1H, $J_{\mathrm{BX}} 4.9$, $\left.J_{\mathrm{AB}} 14.6,1 / 2 \mathrm{CH}_{2}\right), 3.486\left(\mathrm{~s}, 6 \mathrm{H}, \mathrm{OCH}_{3}-2\right.$ and $\left.\mathrm{OCH}_{3}-6\right), 3.748$ (ddd, $1 \mathrm{H}, J$ 2.0, 4.9, 8.2, CH-3), 3.772 (s, 3H, $\left.\mathrm{OCH}_{3}-4\right), 4.688$ (d, 1H, J 2.0, CH-4), 4.969 (q, 1H, J 7.1, 14.1, $\left.\mathrm{CH}_{-} \mathrm{CH}_{3}\right), 5.956(\mathrm{~s}$, 2H, CH-3 and CH-5 Ar), 7.112 (dd, 1H, J 1.5, 8.7, CH-3 Naphth), 7.146 (m, 4H, CH-2, CH-3, CH-5 and CH-6 Ph), 7.224 (t, 1H, J 8.3, CH-4 Ph), 7.390 (s, 1H, CH-1 Naphth), 7.437 (m, 2H, CH-7 and CH-8 Naphth), 7.675 (m, 2H, CH-4 and $\mathrm{CH}-9$ Naphth), 7.794 (dd, 1H, J 2.0, 9.1, CH6 Naphth). ${ }^{13} \mathrm{C}$ NMR $17.50\left(\mathrm{CH}_{3}-\mathrm{CH}\right), 34.48\left(\mathrm{CH}_{2}\right), 50.74(\mathrm{CH}-4), 51.46\left(\mathrm{CH}_{-} \mathrm{CH}_{3}\right), 54.66(\mathrm{CH}-$ 3), $55.21\left(\mathrm{OCH}_{3}-2\right.$ and $\left.\mathrm{OCH}_{3}-6\right), 55.26\left(\mathrm{OCH}_{3}-4\right), 90.23(\mathrm{CH}-3$ and $\mathrm{CH}-5 \mathrm{Ar}), 105.42\left(C_{\text {quat }}-1\right.$ Ar), 125.58 ( $C H-1$ Naphth), 125.71 ( $\mathrm{CH}-8$ Naphth), 125.79 ( $\mathrm{CH}-7$ Naphth), 126.07 (CH-3 Naphth), 127.51 (CH-6 Naphth), 127.86 (CH-9 Naphth), 127.96 (CH-4 Naphth), 128.15 (CH-2 and $\mathrm{CH}-6 \mathrm{Ph}), 128.55(\mathrm{CH}-4 \mathrm{Ph}), 129.15(\mathrm{CH}-3$ and $\mathrm{CH}-5 \mathrm{Ph}), 132.67$ ( $C_{\text {quat }}-5$ Naphth), 133.21 ( $C_{\text {quat }}-10$ Naphth), 137.60 ( $C_{\text {quat }}-2$ Naphth), 138.80 ( $\left.C_{\text {quat }}-1 \mathrm{Ph}\right), 160.19$ ( $C_{\text {quat }}-2$ and $\left.C_{\text {quat }}-6 \mathrm{Ar}\right)$, $161.19\left(C_{\text {quat }}-4 \mathrm{Ar}\right), 170.29(C=\mathrm{O})$.

3-Benzyl-4-(4-(dimethylamino)phenyl)-1-((S)-1-(1-napthyl)ethyl)azetidin-2-one (10) and 3benzyl-4-(4-(dimethylamino)phenyl)-1-((R)-1-(1-napthyl)ethyl)azetidin-2-one (11). The products have the same $\mathbf{R}_{\mathrm{f}}$-values and were isolated as diastereoisomeric mixture; $\mathrm{MS} \mathrm{m} / \mathrm{z}$ 434; HRMS (EI): calcd for $\mathrm{C}_{30} \mathrm{H}_{30} \mathrm{~N}_{2} \mathrm{O}\left(\mathrm{M}^{+}\right)$: 434.2358, found: 434.2329.

(3R,4S)-10 and (3S,4R)-11: $\mathrm{R}_{\mathrm{f}} 0.34\left(\mathrm{CH}_{2} \mathrm{Cl}_{2}\right.$ :acetone 100:1). ${ }^{1} \mathrm{H}$ NMR 1.806 (d, 3H, J 7.1, $\mathrm{CH}_{3^{-}}$ $\mathrm{CH}), 2.868\left(\mathrm{~s}, 6 \mathrm{H}, 2 \times \mathrm{CH}_{3}-\mathrm{N}\right.$ ), 3.057 (A part of ABX, 1H, $J_{\mathrm{AX}} 7.2, J_{\mathrm{AB}} 14.6,1 / 2 \mathrm{CH}_{2}$ ), $3.091(\mathrm{~B}$ part of $\left.\mathrm{ABX}, 1 \mathrm{H}, J_{\mathrm{BX}} 5.6, J_{\mathrm{AB}} 14.6,1 / 2 \mathrm{CH}_{2}\right), 3.313$ (ddd, $\left.1 \mathrm{H}, J 1.9,5.7,7.2, \mathrm{CH}-3\right), 4.005$ (d, 1H, $J$ 1.9, $\mathrm{CH}-4), 5.032$ (q, 1H, J 7.1, 14.0, $\left.\mathrm{CH}-\mathrm{CH}_{3}\right), 6.465$ (dt, 2H, $J$ 1.9, 8.6, CH-2 and $\mathrm{CH}-6 \mathrm{Ar}$ ), 6.809 (dt, 2H, J 2.0, 8.6, CH-3 and $\mathrm{CH}-5 \mathrm{Ar}$ ), 7.049 (d, 1H, J 7.1, CH-2 Naphth), 7.213 (m, 5H, $\mathrm{CH}-2, \mathrm{CH}-3, \mathrm{CH}-4, \mathrm{CH}-5$ and $\mathrm{CH}-6 \mathrm{Ph}$ ), 7.250 (dd, 1H, J 7.1, 8.2, CH-3 Naphth), 7.418 (m, 2H, CH-7 and CH-8 Naphth), 7.627 (d, 1H, J 8.2, CH-4 Naphth), 7.770 (d, 1H, J 7.9, CH-6 Naphth), 7.911 (d, 1H, J 9.0, CH-9 Naphth). ${ }^{13} \mathrm{C}$ NMR $19.72\left(\mathrm{CH}_{3}-\mathrm{CH}\right), 33.88\left(\mathrm{CH}_{2}\right), 40.51\left(\mathrm{CH}_{3}-\mathrm{N}\right)$, $49.89\left(\mathrm{CH}_{-} \mathrm{CH}_{3}\right), 58.74(\mathrm{CH}-4), 59.77(\mathrm{CH}-3), 112.45(\mathrm{CH}-2$ and $\mathrm{CH}-6 \mathrm{Ar}), 122.87(\mathrm{CH}-9$ Naphth), 123.81 ( $C \mathrm{H}-2$ Naphth), 125.52 ( $\mathrm{CH}-7$ or $\mathrm{CH}-8$ Naphth), 125.55 ( $\mathrm{CH}-7$ or $\mathrm{CH}-8$ Naphth), 126.12 ( $\left.C_{\text {quat }}-1 \mathrm{Ar}\right), 126.16$ ( $\left.C \mathrm{H}-4 \mathrm{Ph}\right), 126.55$ (CH-3 Naphth), 127.57 (CH-3 and $C H-5$ Ar), 127.88 (CH-4 Naphth), 128.26 (CH-3 and CH-5 Ph), 128.83 (CH-6 Naphth), 129.43 (CH-2 
and $C \mathrm{H}-6 \mathrm{Ph}), 130.56$ ( $C_{\text {quat }}-10$ Naphth), 133.79 ( $C_{\text {quat }}-5$ Naphth), 136.46 ( $C_{\text {quat }}-1$ Naphth), $138.03\left(C_{\text {quat }}-1 \mathrm{Ph}\right), 150.34\left(C_{\text {quat }}-4 \mathrm{Ar}\right), 170.32(C=\mathrm{O})$.

(3S,4R)-10 and (3R,4S)-11: $\mathrm{R}_{\mathrm{f}} 0.34\left(\mathrm{CH}_{2} \mathrm{Cl}_{2}\right.$ :acetone 100:1). ${ }^{1} \mathrm{H}$ NMR 1.427 (d, 3H, J 7.0, $\mathrm{CH}_{3^{-}}$ $\mathrm{CH}), 2.640\left(\mathrm{~A}\right.$ part of $\left.\mathrm{ABX}, 1 \mathrm{H}, J_{\mathrm{AX}} 8.9, J_{\mathrm{AB}} 15.01 \frac{1}{2} \mathrm{CH}_{2}\right), 2.921\left(\mathrm{~s}, 6 \mathrm{H}, 2 \times \mathrm{CH}_{3}-\mathrm{N}\right), 2.967(\mathrm{~B}$ part of $\left.\mathrm{ABX}, 1 \mathrm{H}, J_{\mathrm{BX}} 5.6, J_{\mathrm{AB}} 15.0,1 / 2 \mathrm{CH}_{2}\right), 3.279$ (ddd, $\left.1 \mathrm{H}, J 1.9,5.6,8.9, \mathrm{CH}-3\right), 3.414(\mathrm{~d}, 1 \mathrm{H}$, $J$ 1.9, CH-4), 5.840 (q, 1H, J 7.0, 13.9, $\left.\mathrm{CH}_{-} \mathrm{CH}_{3}\right), 6.586(\mathrm{dt}, 2 \mathrm{H}, J$ 2.0, 8.6, CH-2 and $\mathrm{CH}-6 \mathrm{Ar}$ ), $6.890(\mathrm{~m}, 4 \mathrm{H}, \mathrm{CH}-3$ and $\mathrm{CH}-5 \mathrm{Ar}$ and $\mathrm{CH}-2$ and $\mathrm{CH}-6 \mathrm{Ph}), 7.016$ (m, 3H, CH-5 and $\mathrm{CH}-6 \mathrm{Ph}$ ), 7.148 (d, 1H, J 7.1, CH-2 Naphth), 7.336 (dd, 1H, J 7.1, 8.1, CH-3 Naphth), 7.516 (ddd, 1H, J 0.8, 6.9, 8.1, CH-7 Naphth), 7.591 (ddd, 1H, J 1.2, 6.9, 8.6, CH-8 Naphth), 7.787 (d, 1H, J 8.1, CH-4 Naphth), 7.879 (d, 1H, J 8.1, CH-6 Naphth), 8.133 (d, 1H, J 8.6, CH-9 Naphth). ${ }^{13} \mathrm{C}$ NMR $18.87\left(\mathrm{CH}_{3}-\mathrm{CH}\right), 34.39\left(\mathrm{CH}_{2}\right), 40.58\left(\mathrm{CH}_{3}-\mathrm{N}\right), 46.55\left(\mathrm{CH}-\mathrm{CH}_{3}\right), 59.00(\mathrm{CH}-3), 60.00(\mathrm{CH}-4)$, 112.21 ( $\mathrm{CH}-2$ and $\mathrm{CH}-6 \mathrm{Ar}), 123.25$ ( $\mathrm{CH}-9$ Naphth), 124.38 (CH-2 Naphth), 124.95 (CH-3 Naphth), 125.93 (CH-7 Naphth), 126.14 ( $\left.C_{\text {quat }}-1 \mathrm{Ar}\right), 126.19$ (CH-4 Ph), 126.88 (CH-8 Naphth), 127.98 (CH-3 and $\mathrm{CH}-5 \mathrm{Ar}), 128.28$ (CH-3 and $\mathrm{CH}-5 \mathrm{Ph}), 128.53$ (CH-2 and $\mathrm{CH}-6 \mathrm{Ph}), 128.64$ (CH-4 Naphth), 128.94 (CH-6 Naphth), 131.23 ( $C_{\text {quat }}-10$ Naphth), 133.83 ( $C_{\text {quat }}-5$ Naphth), 134.39 ( $C_{\text {quat }}-1$ Naphth), 138.26 ( $\left.C_{\text {quat }}-1 \mathrm{Ph}\right), 150.41$ ( $\left.C_{\text {quat }}-4 \mathrm{Ar}\right), 170.04(C=\mathrm{O})$.

3-Benzyl-4-(2,4,6-trimethoxyphenyl)-1-((S)-1-(1-napthyl)ethyl)azetidin-2-one (14) and 3benzyl-4-(2,4,6-trimethoxyphenyl)-1-((R)-1-(1-napthyl)ethyl)azetidin-2-one (15). MS $\mathrm{m} / \mathrm{z}$ 481. HRMS (EI): calcd for $\mathrm{C}_{31} \mathrm{H}_{31} \mathrm{NO}_{4}\left(\mathrm{M}^{+}\right)$: 481.2253, found: 481.2281 .

(3R,4S)-14 and (3S,4R)-15. $\mathrm{R}_{\mathrm{f}} 0.30\left(\mathrm{CH}_{2} \mathrm{Cl}_{2}\right.$ :acetone 100:1). ${ }^{1} \mathrm{H}$ NMR 1.608 (d, 3H, J 7.0, $\mathrm{CH}_{3^{-}}$ $\mathrm{CH}$ ), 3.033 (A part of $\mathrm{ABX}, 1 \mathrm{H}, J_{\mathrm{AX}} 7.6, J_{\mathrm{AB}} 14.6,1 / 2 \mathrm{CH}_{2}$ ), 3.066 (B part of ABX, 1H, $J_{\mathrm{BX}} 5.6$, $\left.J_{\mathrm{AB}} 14.6,1 / 2 \mathrm{CH}_{2}\right), 3.250\left(\mathrm{~s}, 6 \mathrm{H}, \mathrm{OCH}_{3}-2\right.$ and $\left.\mathrm{OCH}_{3}-6\right), 3.654\left(\mathrm{~s}, 3 \mathrm{H}, \mathrm{OCH}_{3}-4\right), 3.733$ (ddd, 1H, J 1.9, 5.6, 7.6, CH-3), 4.918 (d, 1H, J 1.9, CH-4), 5.415 (q, 1H, J 7.0, 13.7, $\left.\mathrm{CH}_{-} \mathrm{CH}_{3}\right), 5.617(\mathrm{~s}, 2 \mathrm{H}$, CH-3 and $\mathrm{CH}-5 \mathrm{Ar}$ ), 6.926 (d, 1H, J 7.1, CH-2 Naphth), 7.039 (t, 1H, J 7.7, CH-3 Naphth), 7.165 (t, 1H, J 8.7, CH-4 Ph), 7.234 (m, 4H, CH-2, CH-3, CH-5 and $\mathrm{CH}-6 \mathrm{Ph}$ ), 7.390 (dd, 1H, J 7.1, 8.1, CH-7 Naphth), 7.475 (dd, 1H, J 7.1, 8.6, CH-8 Naphth), 7.528 (d, 1H, J 8.2, CH-4 Naphth), 7.716 (d, 1H, J 8.1, CH-6 Naphth), 8.250 (d, 1H, J 8.6, CH-9 Naphth). ${ }^{13} \mathrm{C}$ NMR 17.97 $\left(\mathrm{CH}_{3}-\mathrm{CH}\right), 34.62\left(\mathrm{CH}_{2}\right), 46.86\left(\mathrm{CH}_{-} \mathrm{CH}_{3}\right), 49.83(\mathrm{CH}-4), 54.45(\mathrm{CH}-3), 54.98\left(\mathrm{OCH}_{3}-4\right), 55.24$ $\left(\mathrm{OCH}_{3}-2\right.$ and $\left.\mathrm{OCH}_{3}-6\right), 89.96(\mathrm{CH}-3$ and $\mathrm{CH}-5 \mathrm{Ar}), 104.02$ ( $\left.C_{\text {quat }}-1 \mathrm{Ar}\right), 123.43$ (CH-2 Naphth), 123.92 (CH-9 Naphth), 124.76 (CH-3 Naphth), 125.25 ( $\mathrm{CH}-7$ Naphth), 125.90 (CH-8 Naphth), 126.19 (CH-4 Ph), 127.47 (CH-4 Naphth), 128.23 (CH-3 and $C H-5$ Ph), 128.25 (CH-6 Naphth), 129.32 (CH-2 and $C H-6 \mathrm{Ph}), 131.29$ ( $\left.C_{\text {quat }}-10 \mathrm{Naphth}\right), 133.44\left(C_{\text {quat }}-5 \mathrm{Naphth}\right), 135.32\left(C_{\text {quat }}-1\right.$ Naphth), 138.84 ( $\left.C_{\text {quat }}-1 \mathrm{Ph}\right), 159.78$ ( $C_{\text {quat }}-2$ and $\left.C_{\text {quat }}-6 \mathrm{Ar}\right), 160.80$ ( $\left.C_{\text {quat }}-4 \mathrm{Ar}\right), 170.29(C=\mathrm{O})$. (3S,4R)-14 and (3R,4S)-15. $\mathrm{R}_{\mathrm{f}} 0.23\left(\mathrm{CH}_{2} \mathrm{Cl}_{2}\right.$ :acetone 100:1). ${ }^{1} \mathrm{H}$ NMR $1.412\left(\mathrm{~d}, 3 \mathrm{H}, \mathrm{J} 7.0, \mathrm{CH}_{3^{-}}\right.$ $\mathrm{CH}$ ), 2.663 (A part of $\mathrm{ABX}, 1 \mathrm{H}, J_{\mathrm{AX}} 9.6, J_{\mathrm{AB}} 14.8,1 / 2 \mathrm{CH}_{2}$ ), 2.983 (B part of $\mathrm{ABX}, 1 \mathrm{H}, J_{\mathrm{BX}} 5.1$, $\mathrm{J}_{\mathrm{AB}} 14.8,1 / 2 \mathrm{CH}_{2}$ ), 3.551 (s, 6H, $\mathrm{OCH}_{3}-2$ and $\mathrm{OCH}_{3}-6$ ), 3.703 (ddd, $1 \mathrm{H}, J$ 2.0, 5.1, 9.6, $\mathrm{CH}-3$ ), 3.789 (s, 3H, $\left.\mathrm{OCH}_{3}-4\right), 4.274(\mathrm{~d}, 1 \mathrm{H}, J 2.0, \mathrm{CH}-4), 5.734$ (q, 1H, J 7.0, 13.9, $\left.\mathrm{CH}_{-} \mathrm{CH}_{3}\right), 5.963(\mathrm{~s}$, 2H, CH-3 and $\mathrm{CH}-5 \mathrm{Ar}), 6.967$ (dd, 2H, J 1.4, 8.2, CH-2 and CH-6 Ph), 7.018 (t, 1H, J 7.3, CH$4 \mathrm{Ph}$ ), 7.042 (ddd, 2H, J 1.5, 7.3, 8.2, CH-3 and CH-5 Ph), 7.084 (d, 1H, J 7.1, CH-2 Naphth), 7.283 (dd, 1H, J 7.1, 8.2, CH-3 Naphth), 7.506 (ddd, 1H, J 0.9, 6.8, 8.1, CH-7 Naphth), 7.565 
(ddd, 1H, J 1.2, 6.8, 8.4, CH-8 Naphth), 7.763 (d, 1H, J 8.2, CH-4 Naphth), 7.872 (d, 1H, J 8.1, CH-6 Naphth), 8.098 (d, 1H, J 8.4, CH-9 Naphth). ${ }^{13} \mathrm{C}$ NMR $17.12\left(\mathrm{CH}_{3}-\mathrm{CH}\right), 35.01\left(\mathrm{CH}_{2}\right)$, $46.44\left(\mathrm{CH}-\mathrm{CH}_{3}\right), 51.41(\mathrm{CH}-4), 54.66(\mathrm{CH}-3), 55.21\left(\mathrm{OCH}_{3}-2\right.$ and $\left.\mathrm{OCH}_{3}-6\right), 55.25\left(\mathrm{OCH}_{3}-4\right)$, 90.08 ( $C H-3$ and $\mathrm{CH}-5 \mathrm{Ar}), 105.41$ ( $\left.C_{\text {quat }}-1 \mathrm{Ar}\right), 123.25$ (CH-9 Naphth), 124.12 (CH-2 Naphth),

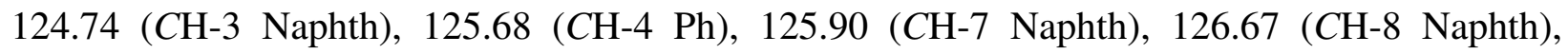
128.06 ( $\mathrm{CH}-3$ and $\mathrm{CH}-5 \mathrm{Ph}), 128.14$ ( $\mathrm{CH}-4 \mathrm{Naphth}), 128.61$ ( $\mathrm{CH}-2$ and $\mathrm{CH}-6 \mathrm{Ph}), 128.67$ (CH-6 Naphth), 131.48 ( $C_{\text {quat }}-10$ Naphth), 133.75 ( $C_{\text {quat }}-5$ Naphth), 134.67 ( $C_{\text {quat }}-1$ Naphth), 139.22 $\left(C_{\text {quat }}-1 \mathrm{Ph}\right), 160.10\left(C_{\text {quat }}-2\right.$ and $\left.C_{\text {quat }}-6 \mathrm{Ar}\right), 161.17\left(C_{\text {quat }}-4 \mathrm{Ar}\right), 170.25(C=\mathrm{O})$.

\section{Pharmacology}

The in vitro antibacterial and antifungal activities of the synthesized compounds against a series of species were determined qualitatively by the agar cup test according to the European Pharmacopoeia. ${ }^{14}$ Suspensions of the test microorganisms were inoculated into sterile melted nutrient agar media and poured into Petri dishes. The bacterial strains were grown in nutrient agar (Serva, Germany) for $24 \mathrm{~h}$ at $37{ }^{\circ} \mathrm{C}$ while the yeast and fungal strains were incubated in yeast peptone dextrose agar (YEPD) and in potato dextrose agar (PDA), respectively for $72 \mathrm{~h}$ at $28{ }^{\circ} \mathrm{C}$. Six wells per dish, each $8 \mathrm{~mm}$ in diameter, were prepared. Fifty microliters of each sample in dimethylsulfoxide $(50 \mathrm{mg} / \mathrm{ml})$ was added to the appropriate well. For pre-diffusion the Petri dishes were placed at $4{ }^{\circ} \mathrm{C}$ for $2 \mathrm{~h}$. The antimicrobial activity was estimated by the diameter of inhibitory zones $(\mathrm{mm})$ in the agar layer. Control experiments were carried out with the pure solvent.

\section{Acknowledgements}

The financial support by the National Research Fund of Bulgaria, Projects UNA-17/2005 and DRNF-02-13/2009, is gratefully acknowledged.

\section{References}

1. Cimarusti, C. M.; Sykes, R. B. Med. Res. Rev. 1984, 4, 1. (b) Beta-Lactam Antibiotics for Clinical Use, S. F. Queener, J. A. Webber, S. W. Queener, Eds.; Clinical Pharmacology, Vol. 4, Marcel Deccer, Inc., New York, 1986. (c) Nathwani, D.; Wood, M. J. Drugs 1993, 45, 866. (d) Saturnino, C.; Buonerba, M.; Martini, S.; Rinaldi, E. R.; Gorga, F.; De Martino, G.; Capasso, A. Biomed. Res. 2004, 15, 100. (e) Singh, G. S.; Mbukwa, E.; Pheko, T. Arkivoc 2007, 80. (f) Wang, Y.; Zhang, H.; Huang, W.; Kong, J.; Zhou, J.; Zhang, B. Eur. J. Med. Chem. 2009, 44, 1638. (g) Keri, R. S.; Hosamani, K. M.; Shingalapur, R. V.; Reddy, H. R. S. Eur. J. Med. Chem. 2009, 44, 5123. (h) Long, B.; He, C.; Yang, Y.; Xiang, J. Eur. J. Med. Chem. 2010, 45, 1181. (i) O’Boyle, N. M.; Carr, M.; Greene, L. M.; Bergin, O.; Nathwani, S. 
M.; McCabe, T.; Lloyd, D. G.; Zisterer, D. M.; Meegan, M. J. J. Med. Chem. 2010, 53, 8569. (j) Mehta, P. D.; Sengar, N. P. S.; Pathak, A. K. Eur. J. Med. Chem. 2010, 45, 5541. (k) Hasan, H.; Akhter, M.; Akhter, W.; Ali, I.; Zaheen, M.; Ahsan, I.; Mahmood, D. Med. Chem. Res. 2011, 20, 1357. (1) O’Boyle, N. M.; Greene, L. M.; Bergin, O.; Fichet, J.-B.; McCabe, T.; Lloyd, D. G.; Zisterer, D. M.; Meegan, M. J. Bioorg. Med. Chem. 2011, 19, 2306. (m) O’Boyle, N. M.; Carr, M.; Greene, L. M.; Keely, N. O.; Knox, A. J. S.; McCabe, T.; Lloyd, D. G.; Zisterer, D. M.; Meegan, M. J. Eur. J. Med. Chem. 2011, 46, 4595. (n) Singh, P.; Sachdeva, S.; Raj, R.; Kumar, V.; Mahajan, M. P.; Nasser, S.; Vivas, L.; Gut, J.; Rosenthal, P. J.; Feng, T.-S.; Chibale, K. Bioorg. Med. Chem. Lett. 2011, 21, 4561. (o) Zarei, M.; Mohamadzadeh, M. Tetrahedron 2011, 67, 5832. (p) Patel, N. B.; Pathak, K. K. Med. Chem. Res. 2011, DOI 10.1007/s00044-011-9728-8.

2. (a) Singh, G. S. Mini Rev. Med. Chem. 2004, 4, 69. (b) Singh, G. S. Mini Rev. Med. Chem. 2004, 4, 93.

3. Romagnoli, M. F.; Fu, K. P.; Neu, H. C. J. Antimicrob. Chemother. 1980, 6, 601.

4. Rodloff, A. C.; Goldstein, E. J.; Torres, A. J. Antimicrob. Chemother. 2006, 58, 916.

5. Baldwin, C. M.; Lyseng-Williamson, K. A.; Keam, S. J. Drugs 2008, 68, 803.

6. Keating, G. M.; Perry, C. M. Drugs 2005, 65, 2151.

7. Keam, S. J. Drugs 2008, 68, 2021.

8. (a) Ojima, I. Acc. Chem. Res. 1995, 28, 383. (b) Ojima, I.; Delaloge, F. Chem. Soc. Rev. 1997, 26, 377. (c) Alcaide, B.; Almendros, P.; Aragoncillo, C. Chem. Rev. 2007, 107, 4437.

9. (a) Hart, D. J.; Ha, D.-C. Chem. Rev. 1989, 89, 1447. (b) The Chemistry of $\beta$-Lactams, Page, M. I., Ed.; Chapman and Hall: London 1992. (c) The Organic Chemistry of $\beta$-Lactams, Georg, G. I., Ed.; VCH: New York 1993. (d) Southgate, R.; Branch, C.; Coulton, S.; Hunt, E. In Recent Progress in the Chemical Synthesis of Antibiotics and Related Microbial Products, G. Lukacs, Ed.; Springer: Berlin, 1993, Vol. 2, 621. (e) De Kimpe, N. In Comprehensive Heterocyclic Chemistry-II; Katritzky, A. R., Rees, C. W., Scriven, E. F. V., Padwa, A., Eds.; Pergamon: Oxford 1996, Vol. 1B, chapter 1.18, pp. 507-589. (f) Tetrahedron 2000, 56, $31^{\text {st }}$ issue was completely devoted to $\beta$-lactam chemistry. (g) Alcaide, B.; Almendros, P. Chem. Soc. Rev. 2001, 30, 226. (h) Singh, G. S. Tetrahedron 2003, 59, 7631. (i) Brandi, A.; Cicchi, S.; Cordero, F. M. Chem. Rev. 2008, 108, 3988. (j) Thompson, S.; Coyne, A. G.; Knipe, P. C.; Smith, M. D. Chem. Soc. Rev. 2011, 40, 4217. (k) Alcaide, B.; Almendros, P. in Progress in Heterocyclic Chemistry, G. W. Gribble, J. Joule, Eds.; Elsevier Ltd., 2011, Vol. 22, Chapter 4, 85.

10. (a) Del Buttero, P.; Molteni, G. Tetrahedron:Asymm. 2006, 17, 1319. (b) Feroci, M. Adv. Synth. Catal. 2007, 349, 2177. (c) Coyne, A. G.; Müller-Bunz, H.; Guiry, P. J. Tetrahedron: Asymm. 2007, 18, 199. (d) Aranda, M. T.; Pérez-Faginas, P.; González-Muñiz, R. Curr. Org. Synth. 2009, 6, 325.

11. (a) Bose, A. K.; Manhas, M. S.; van der Veen, J. M.; Bari, S. S.; Wagle, D. R. Tetrahedron 1992, 48, 4831. (b) C. Palomo, J. M. Aizpurua, I. Ganboa, M. Oiarbide, Eur. J. Org. Chem. 1999, 3223. (c) Magriotis, P. A. Angew. Chem. Int. Ed. 2001, 40, 4377. (d) Palomo, C.; 
Aizpurua, J. M.; Ganboa, I.; Oiarbide, M. Curr. Med. Chem. 2004, 11, 1837. (e) Lee, E. C.; Hodous, B. L.; Bergin, E.; Shih, C.; Fu, G. C. J. Am. Chem. Soc. 2005, 127, 11586. (f) Fu, N.; Tidwell, T. T. Tetrahedron 2008, 64, 10465. (g) A.-R. Zhang, L. He, X. Wu, P.-L. Shao, S. Ye, Org. Lett., 2008, 10, 277-280. (h) Xu, J. Arkivoc 2009, 21. (i) Abraham, C. J.; Paull, D. H.; Dogo-Isonagie, C.; Lectka, T. Synlett, 2009, 1651.

12. (a) Georg, G. I.; Wu, Z. Tetrahedron Lett. 1991, 35, 381. (b) Gunda, T. E.; Sztaricskai, F. Tetrahedron 1997, 53, 7985. (c) Qi, C.-M.; Wang, Y.-F.; Yang, L.-C. J. Heteroc. Chem. 2005, 42, 679-684.

13. (a) Díez, E.; Fernández, R.; Marqués-López, E.; Martín-Zamora, E.; Lassaletta, J. M. Org. Lett. 2004, 6, 2749. (b) Marqués-López, E.; Martín-Zamora, E.; Díez, E.; Fernández, R.; Lassaletta, J. M. Eur. J. Org. Chem. 2008, 2960. (c) Todorov, A. R.; Kurteva, V. B.; Bontchev, R. P.; Vassilev, N. G. Tetrahedron 2009, 65, 10339.

14. European Pharmacopoia, $3^{\text {th }}$ Edition, Deutscher Apotheker Verlag, Stuttgart, 1997, pp. 113. 